\title{
Vulneración del principio de contradicción para abogados ciegos en audiencias del sistema procesal ecuatoriano
}

\section{Violation of the principle of contradiction for blind lawyers in hearings in the Ecuadorian procedural system}

\author{
Jorge Roberto Bravo-Andrade \\ jorgebravosuco@hotmail.com \\ Universidad Católica de Cuenca, Cuenca \\ Ecuador \\ https://orcid.org/0000-0002-3517-1366 \\ Cecilia Ivonne Narváez-Zurita \\ inarvaez@ucacue.edu.ec \\ Universidad Católica de Cuenca, Cuenca \\ Ecuador \\ https://orcid.org/0000-0002-7437-9880 \\ Juan Carlos Erazo-Álvarez \\ jcerazo@ucacue.edu.ec \\ Universidad Católica de Cuenca, Cuenca \\ Ecuador \\ https://orcid.org/0000-0001-6480-2270 \\ Enrique Eugenio Pozo-Cabrera \\ epozo@ucacue.edu.ec \\ Universidad Católica de Cuenca, Cuenca \\ Ecuador \\ https://orcid.org/0000-0003-4980-6403
}

Recibido: 14 de abril de 2020

Revisado: 06 de mayo de 2020

Aprobado: 25 de mayo de 2020

Publicado: 14 de junio de 2020 


\section{RESUMEN}

La Constitución del Ecuador consagra el principio de contradicción aplicable a todos los procesos judiciales. Este principio es garantizado en el momento de alegar y debatir en las audiencias, para lo cual se requiere el completo acceso a la prueba e instrumentos del juicio; esta accesibilidad es limitada para los abogados litigantes ciegos porque el Consejo de la Judicatura no tiene establecido un protocolo, software o herramientas tecnológicas que faciliten este acceso a una persona invidente, dificultando el libre ejercicio de un abogado ciego, por lo tanto, en esta investigación se pretende determinar el grado de vulneración del principio de contradicción a los abogados ciegos en el momento de la objeción de la prueba documental con el fin de propiciar una litigación en igualdad de condiciones para las partes.

Descriptores: Derecho constitucional; derecho al trabajo, procedimiento legal; administración de justicia. (Palabras tomadas del tesauro UNESCO)

\section{ABSTRACT}

The Constitution of Ecuador consecrates the principle of contradiction applicable to all judicial processes. This principle is guaranteed when pleading and debating in the hearings, for which full access to the evidence and instruments of the trial is required; This accessibility is limited for blind litigation lawyers because the Judicial Council does not have established a protocol, software or technological tools that facilitate this access to a blind person, hindering the free exercise of a blind lawyer, therefore, in this investigation It is intended to determine the degree of violation of the principle of contradiction to blind lawyers at the time of the objection of the documentary evidence in order to promote a litigation on equal terms for the parties.

Descriptors: Constitutional law; right to employment; legal procedure; administration of justice. (Words taken from the UNESCO Thesaurus)

\section{INTRODUCCIÓN}

Este estudio se centra en el análisis del principio de contradicción, consagrado en la Constitución de la República del Ecuador. En todas las materias, etapas y diligencias rige la oralidad, este sistema es considerado incluyente porque personas con menor nivel de educación o discapacidad visual, pueden acceder al mismo. Es importante recalcar que el principio de oralidad tiene relación directa con el principio de contradicción ya que lo sustancial en un sistema oral como el que rige en el Ecuador, es el principio de contradicción. Un sistema oral es eminentemente adversarial, en el que se expone y 
debate frente al juez la posición de las partes, las alegaciones mutuas generan la información para que el juzgador pueda emitir pronunciamiento.

En los procesos civiles, penales, administrativos y constitucionales uno de los puntos importantes es la práctica de la prueba, es considerado el momento en el que se argumenta ante un juez la posición de las partes con elementos de evidencia y la contradicción alcanza su punto máximo. El objetivo principal del juez es obtener la verdad, por ello mientras más exigente sea el debate procesal, se obtendrá mejores recursos para dictar una sentencia justa.

Las discapacidades incluyen deficiencias físicas, mentales, intelectuales y sensoriales tales como ceguera, sordera, deterioro de la movilidad y deficiencias en el desarrollo, dificultando o imposibilitando el acceso a diversos espacios. Las deficiencias no están en las personas con discapacidad sino en la sociedad que ha construido un entorno sobre la base de una persona estándar en todos los espacios, incluyendo los laborales. A pesar de existir un cuerpo legal que garantiza los derechos de las personas con discapacidad, se puede evidenciar que el Consejo de la Judicatura no dispone de un mecanismo digital que haga accesible toda la prueba a los abogados ciegos, frente a esta realidad se vulnera el principio de contradicción en un proceso justo.

En el artículo se realizó el análisis teórico del principio de contradicción así como de las garantías establecidas en la constitución para las personas con discapacidad. Con este procedimiento de análisis se pretende analizar la siguiente problemática: ¿Cuáles son las consecuencias de limitar el acceso a la contradicción de la prueba documental a un abogado ciego? Por consiguiente, el objetivo de esta investigación consiste en: determinar el grado de vulneración del principio de contradicción a los abogados ciegos en el momento de la objeción de la prueba documental con el fin de propiciar una litigación en igualdad de condiciones para las partes.

\section{Referencial teórico}

\section{El principio de contradicción y el derecho a la defensa, consagrados en la constitución}

La construcción de la constitución de un país, no solo requiere de procesos jurídicos 
técnicos, sino que además requiere de la organización del Estado y la sociedad. El cuerpo legal que rige un Estado, es la expresión de la cultura, tradiciones, filosofía y pensamiento político de un pueblo; por lo tanto, los derechos fundamentales que los garantizan, constituyen los valores de la sociedad. En este sentido, la Constitución de la República del Ecuador del 2008, establece en el artículo uno que "El Ecuador es un Estado constitucional de derechos y justicia, social, democrático, soberano, independiente, unitario, intercultural, plurinacional y laico. Se organiza en forma de república y se gobierna de manera descentralizada" (Asamblea Nacional, 2008, art.1). Como se puede apreciar, el cuerpo normativo de mayor jerarquía instaura que el Ecuador es un Estado constitucional, garantista de derechos y con administración de justicia, en este contexto, las leyes y códigos deben ser armónicos con las garantías establecidas en la constitución. Es así que, en la administración de justicia el principio de constitucionalidad está sobre el principio de legalidad, por lo tanto, la constitución podrá ser interpretada y aplicada en cualquier caso.

En este orden de ideas, toda nación que se considere un Estado constitucional de derechos debe garantizar la aplicación del debido proceso. Es importante reconocer que la existencia de esta garantía demuestra un avance civilizado que tomó siglos conseguirlo ya que desde el momento que la humanidad decidió organizarse en comunidades, se establecieron reglas de convivencia, dando énfasis al juzgamiento de actos incorrectos. Al paso del tiempo, se registran diversos momentos históricos que contribuyeron a la construcción de la definición actual de debido proceso.

Al respecto, (Ferrer, 2016) afirma que el debido proceso es un derecho que tiene alcances generales por lo que busca de forma justa dar solución a controversias expuestas ante autoridades judiciales, a efectos de proteger los derechos legales que posee una persona y asegurar justicia. Esta última, es una fuente que deriva un conjunto de derechos procesales que no forman parte de manera exclusiva de la norma ya que dependen también de la interpretación que se realice de la misma sobre situaciones concretas.

En términos específicos, se puede afirmar que el debido proceso, corresponde a un cúmulo de derechos y garantías que buscan proteger a personas de los posibles excesos 
o riesgos de abuso por parte del Estado, para lo cual deberá observarse las garantías básicas sin menoscabo de otras que establezca el marco constitucional (Vaca-Andrade, 2010).

El debido proceso es un instrumento que se instauró para proteger a los ciudadanos de los abusos de las autoridades del Estado, emanando principios fundamentales como es el principio de contradicción. En el Ecuador rige el sistema oral, el mismo que es considerado un sistema garantista de derechos y generador de transparencia en los procesos que se ejecuten. La oralidad en las audiencias, genera la posibilidad de que las partes sean escuchadas en igualdad de condiciones a diferencia del proceso inquisitivo, en el cual se dictaba una sentencia con la revisión de un expediente y sin presencia de las partes. Este sistema se caracteriza por ser más incluyente ya que al ser oral, las personas con niveles de educación más bajos o con discapacidad visual pueden acceder con mayor facilidad a los instrumentos del proceso.

Cabe considerar, por otra parte, que un elemento sustancial de un sistema oral, es el principio de contradicción, ya que es un sistema adversarial, en el que se expone y debate frente al juez la posición de las partes. La finalidad del proceso, es llegar a la verdad procesal, contexto que genera una exigencia en la contradicción de las partes, mientras más debate o elementos tenga el juez, más certero será su dictamen, "del carácter contradictorio de la relación procesal, surge la trascendental etapa en el proceso que lleva a las partes en forma de juicio oral que permite alegaciones mutuas" (ReinosoHermina, 2001, pág. 72). Estas alegaciones mutuas generan la información para que el juez pueda dictar la sentencia.

La garantía constitucional de un debido proceso implica la existencia del principio de inocencia de toda persona, razón por la cual existe el derecho a la defensa. Por lo tanto, el principio de contradicción es sustancial en el ejercicio de una defensa adecuada, para expresar la oposición a la contra parte y a los elementos probatorios que existen en el proceso; la oralidad es el mecanismo para la proporción de la información, este objetivo se lo puede alcanzar siempre que se permita la contradicción, es decir, cuando las partes lleguen a debatir sobre las posiciones del uno o del otro, ya que toda persona debe ser 
escuchada. La existencia de una defensa amplia, demuestra que existió un debido proceso. La doctrina establece que se debe tener una igualdad de armas, para que sea una justa contradicción.

En este sentido, (Zavala-Baquerizo, 2002), considera que el principio de contradicción de la prueba, comprende un derecho garantizado por la ley a las partes, ya que las partes tienen derecho a conocer, criticar y oponerse a la prueba con el objetivo de desvalorizar a la otra parte. Uno de los puntos más importantes de todo proceso es la presentación de la prueba ya que es el momento en el que se argumenta al juez la posición de las partes con elementos de evidencia, bajo este contexto, el autor señala que es un derecho de las partes tener una posición frente la prueba presentada.

Por otro lado, (Guerrero-Vivanco, 2004) sostiene que el principio de contrariedad constituye un debate entre las partes con el objetivo de que la prueba de cargo sea rebatida por la prueba de descargo. Es decir, en este momento del proceso, es necesario o se requiere el debate de lo presentado. Los dos autores coinciden que la contradictoriedad, es un derecho que se debe ejercer en el momento de presentar la prueba con el que se garantiza el debate de las partes frente al juez para exponer sus posiciones.

Sin duda, la contradicción alcanza su punto más alto del debate, en la audiencia en la que se valora la actividad probatoria y se permite la oposición y controversia que hace la crítica a la crítica de la parte opositora (Zavala-Baquerizo, 2002). En el proceso de valoración de la prueba, es el momento en que se forman los criterios para la toma de una resolución, esta certeza solo puede lograrse con el principio de contradicción.

A este respecto, (Vaca-Andrade, 2010) sostiene que la prueba, y todos los elementos del juicio, deben ser notificados oportunamente, ya que este principio garantiza que las partes procesales tengan la misma oportunidad de conocer, rebatir y discutir las pruebas, bajo este contexto, las pruebas de último momento o secretas no serán válidas por no haber sido notificadas con anticipación.

El principio de contradicción, está más orientado a beneficio del accionado, ya que es la parte interesada a que se lleve a cabo un debido proceso. Para ejercer este principio, se 
requiere tener el derecho a la defensa, por lo tanto, la contradicción no solo es un principio, sino también un derecho; a través del cual las partes presentan sus argumentos y pruebas así como también cuestionan las pruebas de la otra parte. Para (Quezada, 2008) el principio de contradicción consiste en reconocer y aceptar en proceso judicial, la posibilidad efectiva de que las partes puedan comparecer a fin de que prevalezcan sus respectivas pretensiones. Uno de los derechos de las partes es ser reconocidas en el proceso de orden judicial con sus propias aportaciones para expresar sus posiciones. EI principio de contradicción se deberá aplicar en todas las etapas del proceso, por lo tanto, la contrariedad también debe existir en la fase preprocesal, en la construcción de las pruebas.

\section{Vulnerabilidad de derechos hacia las personas con discapacidad visual}

De acuerdo a la (Organización Mundial de la Salud, 2020) la discapacidad comprende las restricciones de participación, limitaciones de la actividad y deficiencias, las últimas se entienden como problemas que inciden en la función corporal; las limitaciones son dificultades que tienen las personas para ejercer tareas o acciones, en tanto que, las restricciones de la participación representan complicaciones para participar en situaciones vitales. La definición de la capacidad física, explica que una deficiencia limita o impide el desarrollo de una actividad a una persona. En contraste, se presenta la definición de discapacidad, desde un enfoque social,

Como una desventaja o restricción para una actividad que es causada por una organización social contemporánea que toma poca o nada en cuenta a las personas que tienen deficiencias físicas (sensoriales o mentales) y de esa manera las excluye de la corriente principal de las actividades sociales. (Brogna, 2006, parr.2)

En este contexto, se puede determinar que las estructuras sociales están construidas de tal manera que generan obstáculos para el libre desarrollo en igualdad de condiciones de todas las personas (Gutierrez Mejía, Narváez Zurita, Borja Pozo, \& Erazo Álvarez, 2020). La discapacidad es considerada una condición individual, procedente de una tragedia personal, ocasionada por el déficit de alguna característica física o mental "los sujetos no son considerados responsables de su condición, pero sí de hacer todo lo posible por 
recuperar la salud en orden a funcionar lo más normalmente posible y a «acomodarse» subjetivamente a su condición de discapacitado" (Gancio, y otros, 2009, p.9), lo que se establece como discapacidad, es una condición fuera de lo normal, por lo tanto, no podrá adaptarse a lo estandarizado y son sometidos a terapias y procedimientos para que logren lo estandarizado.

En la sociedad moderna existe una caracterización de las condiciones, por tanto, entender a la discapacidad como déficit y como un problema biológico, donde aparece de manera marcada el cuerpo normal o anormal, deficiente o eficiente, que es útil en la vida productiva o no, el que está dentro o fuera del estándar; deviene de una ideología de la normalidad (Mendoza \& Espinoza, 2016). A pesar de que existen cuerpos legales a nivel internacional y nacional que garantice los derechos de las personas con discapacidad; la sociedad y el sistema por los paradigmas construidos vulneran los derechos de las personas con discapacidad ya que es un sujeto que no está categorizado como estándar.

Una de las propuestas más retadoras para los Estados, consiste en crear fuentes de empleo y generar oportunidades laborales para las personas con discapacidad, así como darles apoyo para la búsqueda, obtención, mantenimiento del empleo y retorno al mismo, como también, supervisar que en las distintas plazas de trabajo se realicen adaptaciones razonables en la infraestructura para las personas con discapacidad. Sin embargo, en franca contradicción, el derecho a trabajar de este grupo se encuentra limitado en la actualidad. Frente a esta realidad se enfrentan los abogados que tienen una visión limitada o carecen completamente de ella.

Para tener una eficiente administración de justicia, se requiere contar con un debido proceso; en el Ecuador el sistema procesal es el medio para la realización de la justicia, el mismo que consagra a la oralidad aplicable en todas sus materias, etapas y diligencias, y un principio fundamental como es el de contradicción. Este principio llega a su máxima expresión, dentro de un proceso, en la audiencia en el momento del debate y objeción de la prueba, no obstante, al no tener una accesibilidad de todas las pruebas por parte de los abogados ciegos se vulnera el principio de contradicción así como el derecho del 
ejercicio profesional de una persona con discapacidad visual.

\section{Violación del principio de contradicción en un proceso judicial a los abogados litigantes ciegos}

En el Ecuador, de acuerdo a (CONADIS, 2019), están registrados 485.325 personas con discapacidad, de las cuales el $11,66 \%$ tienen una discapacidad visual, lo que representa 56.570 personas. Se encuentran registradas 32.587 personas con discapacidad visual que están entre 19 y 65 años (PEA), de las cuales 9.409 personas, están laboralmente activas. El $28.9 \%$ de las personas registradas en el CONADIS que tienen discapacidad visual y están en un rango de edad entre 19 y 65 años, han logrado obtener un trabajo.

Al revisar información sobre la condición de abogados litigantes ciegos, no se encuentra material de investigación sobre la condición de estos profesionales en su libre ejercicio. El Consejo de la Judicatura del Ecuador dispone de un manual de atención en derechos de personas con discapacidad en la función judicial, el mismo que está enfocado a la atención de personas con discapacidad con la perspectiva de usuarios así como la profundización de los derechos consagrados para las personas con discapacidad.

El (Consejo de la Judicatura, 2015) sostiene que "en el marco del modelo social y de derechos humanos, con el fin de precautelar el derecho de las personas con discapacidad a la participación plena y efectiva en la sociedad, es necesario adoptar ajustes razonables que les permitan estar incluidos en la sociedad y no fuera de ella", la institución hace énfasis en ajustes razonables, es decir, que se podrán generar condiciones que estén accesibles; no establece protocolos específicos para generar la posibilidad de litigio a un abogado ciego, ni dispone de un software o tecnología que pueda generar la visualización de documentos para no videntes.

Una de las recomendaciones que establece el manual es que "se deben identificar los lenguajes alternativos $\mathrm{y} / \mathrm{o}$ aumentativos de las personas con discapacidad sensorial para ser aplicados en el proceso y así estas puedan participar de manera directa a través de los medios pertinentes para que exista la interacción con las y los operadores de justicia" (Consejo de la Judicatura, 2015) el manual establece recomendaciones que deben seguir 
Jorge Roberto Bravo-Andrade; Cecilia Ivonne Narváez-Zurita; Juan Carlos Erazo-Álvarez; Enrique Eugenio Pozo-Cabrera

los operadores de justicia si tuvieran un caso de discapacidad, está enfocado al acceso estructural, comunicacional y legal. Las recomendaciones son generales de acciones que se deberían improvisar en ese momento, más no existe capacitaciones a los operadores de la justicia en atención y manejo a personas con discapacidad, las acciones que deben generar son intuitivas más que técnicas.

Para (Espinoza-Mina \& Gallegos-Barzola, 2018) "El Estado define políticas públicas que están dirigidas a solucionar problemas que agobian a la población en general o una parte de ellas, en este caso al sector vulnerable de los discapacitados; empero como en todo proceso, existen fallas o limitantes que impiden que se cumplan por completo las metas que fueron establecidas", en este caso se puede evidenciar la falta de investigación de las necesidades de los abogados ciegos por parte del Consejo de la Judicatura, para implementar protocolos, herramientas y tecnología acorde a su necesidad.

Las pruebas son actuadas de manera oral o escrita en una audiencia, a pesar de que el sistema oral es más incluyente aún, existen instrumentos escritos o imágenes que son consideradas como prueba. Para un abogado litigante ciego, el no disponer de formatos que sean accesibles para su discapacidad, dificulta la posibilidad de debate de la prueba y con ello el ejercicio del principio de contradicción.

Esta condición genera la necesidad de contar con personal de apoyo para llevar a cabo su libre ejercicio, como es el caso del Doctor Amado Vicente León, quien en una entrevista sostiene "como personas nos puede faltar una pierna, un brazo o alguna otra parte pero la visión es mucho más porque es clave principal para el ser humano, sin embargo he sido responsable, fuerte y tengo el mejor equipo de colaboradores con los que hemos defendido algunos procesos en los tribunales" (Crónica, 2016). En otros casos las personas con discapacidad visual que han podido culminar sus estudios superiores en leyes, han visto imposibilitado su libre ejercicio por lo que realizan otro tipo de actividades.

\section{MÉTODO}

El presente estudio se realizó bajo una revisión exhaustiva de la literatura publicada en 
relación al principio de contradicción para abogados ciegos, este proceso inició con una revisión automatizada mediante la identificación de descriptores o palabras clave en bases de datos jurídicas, además, se realizó una revisión documental en revistas y cuerpos jurídicos para sustentar la problemática en estudio. En el proceso de levantamiento de datos se extrajo la información más sobresaliente de autores reconocidos a nivel nacional e internacional, tratando de evitar sesgos y prejuicios que influyan en las conclusiones finales.

Por lo expuesto, la investigación se desarrolló bajo un enfoque cualitativo, con alcance descriptivo y finalidad transversal, no se utilizaron fuentes primarias de datos para sustentar las ideas expuestas en la investigación, por lo tanto, las técnicas de análisis de información se respaldaron en el recuento histórico y revisión bibliográfica de las variables en estudio. En consecuencia, este artículo se centra en presentar de forma ordenada, coherente y lógica, los elementos que lograron su validación empírica en trabajos anteriores y que han mostrado en el trascurso del tiempo que a nivel de los procesos de audiencia llevados a cabo en Ecuador existe vulneración en el principio de contradicción a los abogados ciegos en el momento de la objeción de la prueba documental, lo que deja en evidencia una litigación en desigualdad de condiciones para las partes.

En cuanto a los métodos empleados, el analítico - sintético permitió condensar y extraer la información analizada sobre el principio de contradicción, la litigación en igualdad de condiciones y la vulneración de los derechos de los abogados ciegos durante las audiencias en el sistema procesal, para ello fue necesario establecer un punto de vista interpretativo y evaluativo de la información disponible.

\section{DISCUSIÓN}

Los cuerpos adjetivo legales vigentes en el Ecuador son sujetos a un debido proceso, que tiene como garantía básica y principio relevante la contradicción, esta llega a su máxima expresión en las audiencias, que es el momento procesal en el que las partes son escuchadas en igualdad de condiciones, y cuando el juzgador recibe la mayor 
cantidad de información para tomar una resolución justa.

La discapacidad tiene dos enfoques para su concepción la física y la social. La discapacidad social genera mayores limitaciones ya que todo el sistema está construido con la percepción de una persona estándar por lo que una persona con discapacidad tiene que hacer el esfuerzo para tratar de ser lo más "normal" posible.

Los avances en derechos y garantías para las personas con discapacidad, en las últimas décadas han sido representativos y se han logrado expresar en instrumentos legales a nivel nacional e internacional. A pesar de existir normativas avanzadas, las políticas públicas no llegan a ser tan efectivas ya que se limitan a construirlas sin entender las verdaderas necesidades de este grupo, no existe un conocimiento exacto de los instrumentos y condiciones requeridas para un desenvolvimiento óptimo de una persona con discapacidad.

No disponer de investigaciones que revelen las limitaciones de un abogado litigante ciego para ejercer su profesión dentro del órgano rector de justicia, evidencia el poco asesoramiento técnico que brinda el Consejo de la Judicatura para el correcto desenvolvimiento de un profesional con discapacidad visual en un proceso judicial.

Para que un abogado litigante pueda ejercer a plenitud el principio de contradicción, se requiere del completo acceso a la prueba; la misma que se presentará de forma oral o escrita. No se establece un procedimiento, un software o tecnología que garantice que todos los elementos presentados en un proceso judicial serán de acceso pleno para un abogado ciego, razón por la cual, al no tener acceso a la totalidad de los elementos del juicio, se viola el principio de contradicción ya que se ve limitado su posibilidad de litigar en igualdad de condiciones.

Esta dinámica ocasiona que para el libre ejercicio de un profesional del derecho, se requiera de un equipo de apoyo en los procesos, de lo contrario se imposibilita su libre ejercicio. 


\section{FINANCIAMIENTO}

No monetario.

\section{AGRADECIMIENTO}

A la Universidad Católica de Cuenca por apoyar la investigación.

\section{REFERENCIAS CONSULTADAS}

Asamblea Nacional. (20 de Octubre de 2008). Constitución de la República del Ecuador 2008 [Constitution of the Republic of Ecuador 2008]. Quito: Asamblea Nacional. Obtenido de https://n9.cl/xo9c

Brogna, P. (2006). "La discapacidad un obra escrita por los autores por los actores de reparto" el paradigma de la discapacidad: Realidad o Utopía en el nuevo escenario Latinoamericano,["Disability a work written by the authors by the supporting actors" the paradigm]. México: Universidad Nacional Autónoma de México. Obtenido de https://n9.cl/0plod

CONADIS. (2019). Consejo Nacional para la Igualdad de Discapacidades. Recuperado el 29 de Mayo de 2020, de Consejo Nacional para la Igualdad de Discapacidades: https://www.consejodiscapacidades.gob.ec/estadisticas-de-discapacidad/

Congreso Nacional. (13 de Enero de 2009). Código de procesamiento penal [Criminal Processing Code]. Quito. Obtenido de https://n9.cl/q2vfu

Consejo de la Judicatura. (2015). Manual de Atención en derechos de personas con discapacidad en la función judicial, [Manual of Attention in rights of people with disabilities in the judicial function]. Ecuador: Consejo de la Adjudicatura. Obtenido de https://n9.cl/c0n9

Crónica. (15 de Julio de 2016). León: 20 años como abogado pese a su discapacidad visual [Leon: 20 years as lawyer in spite of your visual disability]. Crónica, pág. 1. Obtenido de https://n9.cl/ika8

Espinoza-Mina, M. A., \& Gallegos-Barzola, D. d. (2018). Inserción laboral de las personas con discapacidad en Ecuador,[Labor insertion of people with disabilities in Ecuador]. Espacios, 51. Obtenido de https://n9.cl/i564 
Jorge Roberto Bravo-Andrade; Cecilia Ivonne Narváez-Zurita; Juan Carlos Erazo-Álvarez; Enrique Eugenio Pozo-Cabrera

Ferrer, E. (2016). Panorámica del Derecho Procesal Constitucional y Convencional,[Overview of Constitutional and Conventional Procedural Law]. México: Instituto de Investigaciones Jurídicas, UNAM.

Gancio, E., Escudero, B., García, A., Martínez, E., Miguez, M., Moreira, C., Pintos, A. (2009). El papel de la ideología de la normalidad en la producción de discapacidad,[The role of the ideology of normality in the production of disability]. Buenos Aires: Universidad Nacional de Entre Ríos. Obtenido de https://www.redalyc.org/pdf/145/14512426004.pdf

Guerrero-Vivanco, W. (2004). Derecho Procesal Penal,[Criminal Procedural Law]. Ecuador: Pudeleco Editores.

Gutierrez Mejía, F., Narváez Zurita, C., Borja Pozo, C., \& Erazo Álvarez, J. (2020). La igualdad y no discriminación de las personas con discapacidad intelectual en la educación superior,[equality and non-discrimination of people with intellectual disabilities in higher education]. Iustitia Socialis, 5(1), 5. Obtenido de https://n9.cl/sy8qx

Mendoza, C., \& Espinoza, R. (2016). La construcción social sobre la discapacidad y su correlación en la poca producción de acceso al uso de los medios audiovisualez [Social construction on disability and its correlation in access]. México: Universitdad Autónoma del Estado de México. Obtenido de https://n9.cl/0a0u

Organización Mundial de la Salud. (20 de Enero de 2020). Organización Mundial de la Salud, [World Health Organization]. Obtenido de Organización Mundial de la Salud, [World Health Organization]: https://n9.cl/61c4

Quezada, G. (2008). El proceso penal: Principios Fundamentales, [The criminal process: Fundamental Principles]. Buenos Aires: Astrea.

Reinoso-Hermina, A. (2001). El juicio acusatorio oral en el nuevo código de procedimiento penal ecuatoriano,[The oral accusatory trial in the new Ecuadorian criminal procedure code]. Quito: Progama Nacional de apoyo a la reforma de la administración de justicia del Ecuador Projusticia.

Vaca-Andrade, R. (2010). Manual de derecho procesal penal, tomo I.[Manual of criminal procedural law, volume I]. Quito: Corporación de Estudios y Publicaciones. 
Jorge Roberto Bravo-Andrade; Cecilia Ivonne Narváez-Zurita; Juan Carlos Erazo-Álvarez; Enrique Eugenio Pozo-Cabrera

Vallejos, I. (2009). La categoría de la normalidad: una mirada sobre viejas y nuevas formas de disciplinamiento social,[The category of normality: a look at old and new forms of social discipline]. En A. Rosato, \& M. Angelino, Discapacidad e ideología de la normalidad: Desnaturalizar el déficit (págs. 95-115). Buenos Aires: Noveduc. Obtenido de https://n9.cl/dipi

Zavala Baquerizo, J. (2004). Trato de derecho procesal penal, [Treatment of criminal procedural law]. Guayaquil: EDINO.

Zavala-Baquerizo, J. (2002). El debido proceso penal, [Due process of law]. Guayaquil: Edino. 